\title{
Video Article \\ Methods for Precisely Localized Transfer of Cells or DNA into Early Postimplantation Mouse Embryos
}

\author{
Yali Huang ${ }^{1}$, Ron Wilkie ${ }^{1}$, Valerie Wilson ${ }^{1}$ \\ ${ }^{1}$ MRC Centre for Regenerative Medicine, School of Biological Sciences, University of Edinburgh
}

Correspondence to: Yali Huang at yali.huang@ed.ac.uk

URL: https://www.jove.com/video/53295

DOI: doi:10.3791/53295

Keywords: Developmental Biology, Issue 106, Grafting, electroporation, postimplantation, mouse, epiblast stem cell, EpiSC, embryo culture

Date Published: $12 / 25 / 2015$

Citation: Huang, Y., Wilkie, R., Wilson, V. Methods for Precisely Localized Transfer of Cells or DNA into Early Postimplantation Mouse Embryos. J. Vis. Exp. (106), e53295, doi:10.3791/53295 (2015).

\section{Abstract}

Manipulation and culture of early mouse embryos is a powerful yet largely under-utilized technology enhancing the value of this model system. Conversely, cell culture has been widely used in developmental biology studies. However, it is important to determine whether in vitro cultured cells truly represent in vivo cell types. Grafting cells into embryos, followed by an assessment of their contribution during development is a useful method to determine the potential of in vitro cultured cells. In this study, we describe a method for grafting cells into a defined site of early postimplantation mouse embryos, followed by ex vivo culture. We also introduce an optimized electroporation method that uses glass capillaries of known diameter, allowing precise localization and adjustment of the number of cells receiving exogenous DNA with both high transfection efficiency and low cell death. These techniques, which do not require any specialized equipment, render experimental manipulations of the gastrulation and early organogenesis-stage mouse embryo possible, allowing analysis of commitment in cultured cell subpopulations and the effect of genetic manipulations in situ on cell differentiation.

\section{Video Link}

The video component of this article can be found at https://www.jove.com/video/53295/

\section{Introduction}

Cell culture has been widely used in developmental biology studies. Mouse embryonic stem cells (ESCs) and epiblast stem cells (EpiSCs) can differentiate into all three germ layers in vitro and are a useful model for cell differentiation in early mammalian embryogenesis. The derivation of these cell lines has opened up an opportunity for in vitro manipulation and detailed investigation of the localized signaling events and transcriptional networks operating during early embryonic patterning. However, it remains important to determine the in vivo relevance of any manipulations performed in culture. The in vivo potential of preimplantation embryo-derived mouse ESCs has been assessed by introducing them back into preimplantation embryos (morulae or blastocysts) ${ }^{1}$. However, EpiSCs that represent the epiblast cells in postimplantation embryos cannot integrate efficiently in preimplantation embryos ${ }^{2,3}$. Our previous findings have shown that EpiSCs can efficiently generate chimeras and contribute to all germ layers, when grafted into postimplantation embryos ${ }^{4}$. Thus, the best way to evaluate the in vitro cultured cells is to introduce them to their corresponding environment in vivo.

Electroporation is a widely used method to deliver exogenous molecules into targeted cells in both in vivo and in vitro experiments. Electrical energy can generate a large number of pores in the cell membrane, which allows exogenous deoxyribonucleic acid (DNA) or ribonucleic acid (RNA) to enter the cells. One of the biggest challenges for this technique is to combine optimal cell viability with high electrotransfection efficiency ${ }^{5,6}$. For electroporation of nucleic acids in embryonic tissues, gold plated electrodes have most commonly been used, allowing targeting of cells in a broad spatial range ${ }^{7-9}$. To achieve a more localized gene transfer, a needle-shaped electrode has been utilized to achieve a focal electric field ${ }^{10,11}$. Using this method, the authors showed that after the electroporation, around 30-60 cells had taken up the DNA construct ${ }^{11}$. Nevertheless, it seems that accurately adjusting the number of electroporated cells remains difficult with a fixed-width electrode. The capillary electroporation technique has been used to deliver plasmids to single cells ${ }^{12-14}$. However, this technique has not been applied for electroporating plasmids to embryos ex vivo. More recently, a microdevice has been reported to locally electroporate a few distal visceral endoderm cells (less than 4 cells) in early postimplantation mouse embryos ${ }^{15}$. However, it is still unknown whether this device can efficiently target ectoderm and mesoderm ex vivo.

In this study we describe two novel methods to assess cellular and gene function in early post-implantation embryos. We first demonstrate how to graft in vitro cultured cells into defined sites in early mouse embryos to assess their in vivo potential. The integration of the grafted cells and their descendants, all labelled by a genetic tag (e.g., a green fluorescent protein (GFP), can be further examined by immunostaining of tissue specific proteins ${ }^{4}$. Secondly, we describe an improved method to precisely deliver DNA to localized sites in the embryo via electroporation. Rather than using a needle-shaped electrode, we inserted a thin wire inside a fine-tipped glass capillary, and demonstrate that this modification can deliver DNA to a small number of cells with high efficiency and limited cell death. Moreover, we show that by using glass capillaries with 
different opening sizes, we can control the number of electroporated cells. Therefore, we believe this method can be of great use to study early embryonic patterning involving small numbers of cells.

\section{Protocol}

All animal experiments were carried out in accordance with UK Home Office Regulations as specified in the Animals (Scientific Procedures) Act (1986) under Project Licence number 60/4435. To collect embryos at specific developmental stages, timed matings were set up O/N. Noon on the day of finding a vaginal plug was designated embryonic day (E) 0.5 .

\section{Dissecting the E7.5 or E8.5 Postimplantation Embryos for Ex Vivo Culture}

1. Sacrifice the pregnant female mice by cervical dislocation.

2. Isolate the uterus using scissors, holding it with forceps, and place in a $30 \mathrm{~mm}$ dish filled with $\mathrm{M} 2$ medium.

3. Carefully tear apart the myometrium using two pairs of fine forceps.

4. Peel away the decidua, being careful not to puncture the extraembryonic cavities.

5. Remove the Reichert's membrane by pinching it with forceps and slowly separating it from the embryo.

6. Check the embryos under a dissecting stereomicroscope to ensure that the yolk sac, amnion and ectoplacental cone are intact.

7. Transfer the embryos to a clean dish of M2 using a pipette and place on a $30 \mathrm{~mm}$ plastic Petri dish lid on ice (the 'ice platform') to partially chill embryos.

8. If required, store embryos in $\mathrm{M} 2$ on an ice platform for up to $1.5 \mathrm{hr}$, e.g., during media preparation or manipulation of small batches of $\sim 3-4$ embryos at RT. Note: Recovery and dissection of rodent embryos has been described in detail previously $y^{7,8,16}$

\section{Prepare the Embryo Culture Medium}

1. Freshly thaw either commercially-available rat serum (see Glanville-Jones et al. ${ }^{13}$ for specifications), or rat serum prepared in-house according to Copp and Cockroft ${ }^{16}$ that was heat inactivated for 30 min at $56{ }^{\circ} \mathrm{C}$ and frozen in $1 \mathrm{ml}$ aliquots at $-80^{\circ} \mathrm{C}$

Note: Commercially-available rat serum is acceptable for culture periods of $24-36 \mathrm{hr}$, although serum prepared in-house is, in our experience, superior for culture periods up to $48 \mathrm{hr}$.

2. Freshly prepare an excess (e.g., $10 \mathrm{ml}$ ) of defined supplements consisting of Glasgow Minimum Essential Medium (GMEM), $1 \%$ NonEssential Amino Acids (NEAA), 2 mM L-glutamine and $1 \mathrm{mM}$ sodium pyruvate.

3. Calculate the volume of culture medium that is needed according to the number of embryos of each stage that are going to be cultured (see section 3). Mix the rat serum with the defined supplements to make up $50 \%$ culture medium (1:1 (v/v) rat serum:defined supplements) and/or $75 \%$ culture medium ( $3: 1(\mathrm{v} / \mathrm{v})$ rat serum: defined supplements).

4. Pass the embryo culture medium through a $0.45 \mu \mathrm{m}$ filter and add $10,000 \mathrm{I} . \mathrm{U} / \mathrm{ml}$ penicillin and $10 \mu \mathrm{g} / \mathrm{ml}$ streptomycin Note: Freshly thawed L-glutamine and sodium pyruvate solution is crucial for embryo development during ex vivo culture.

\section{Ex Vivo Culture Conditions for Different Embryonic Stages}

1. For E7.5 embryos: static culture using 4-well plates in an incubator supplied with $5 \% \mathrm{CO}_{2}$ in air at $37{ }^{\circ} \mathrm{C}$ for 24 hr. Culture up to two embryos per well in $1 \mathrm{ml}$ of $50 \%$ culture medium.

2. For E8.5 embryos: use a roller culture apparatus ${ }^{8}$ rotating at $35 \mathrm{revs} / \mathrm{min}$ incorporating continuous gassing with $5 \% \mathrm{CO}_{2}$ in air at $37{ }^{\circ} \mathrm{C}$ for 24 $\mathrm{hr}(1 \mathrm{ml}$ of $50 \%$ culture medium per embryo).

3. For E9.5 embryos: use a roller culture apparatus rotating at 35 revs/min incorporating gases supplied with $5 \% \mathrm{CO}_{2}, 40 \% \mathrm{O}_{2} 55 \% \mathrm{~N}_{2}$ at $37{ }^{\circ} \mathrm{C}$ for $24 \mathrm{hr}$ ( $1 \mathrm{ml}$ of $75 \%$ culture medium per embryo).

Note: Culture embryos within $3 \mathrm{hr}$ after euthanizing the mice as prolonged periods in M2 adversely affect development. See Copp and Cockroft ${ }^{16}$ for the method of ex vivo embryo culture.

\section{Grafting Cultured Cells into E7.5 or E8.5 Mouse Embryos}

1. Physically scrape EpiSCs, which ubiquitously express GFP, from a 6-well culture plate using a 20-200 $\mu$ pipette tip and place them in a 30 $\mathrm{mm}$ dish containing the embryos

Note: To insert cell clumps in the embryos, cells should be physically scrapped rather than trypsinized.

2. Attach a hand-pulled grafting capillary to the aspirator tube to make a mouth pipette.

3. Gently suck the mouth pipette to draw one or more cell clumps of size $>20$ cells into the grafting capillary.

4. Gently blow out the cells, to partially disperse large clumps.

5. Select one cell clump containing $\sim 10-20$ cells and suck into the grafting capillary again, keeping it close to the opening of the capillary. Be careful not to move the cell clump in and out of the capillary repeatedly, to avoid break-up into smaller pieces.

6. Hold the embryo loosely in place with a pair of forceps and insert the grafting capillary into the region of interest to create an opening

7. Gently expel the clump out of the grafting capillary, leaving the short string of 10-20 cells lodged in the embryo.

8. Repeat the grafting procedure for the desired number of embryos. Use batch sizes of 3-4 embryos for convenience.

9. Leaving embryos in the same dish of M2 medium, image the grafted embryos using a fluorescence compound dissecting microscope with camera, keeping imaging time to a minimum to avoid exposure of the embryos to excessive light and heat.

Note: Determine imaging times empirically as these will depend on the specifications of the camera and microscope, as well as the nature and fluorescence intensity of the fluorophore. 
10. Transfer the embryo in a pastette with a minimal volume of $\mathrm{M} 2$ medium to pre-equilibrated culture medium (see section 3 ) immediately after imaging.

Note: Carefully examine the morphology of the embryos after grafting. Only culture the intact embryos.

\section{Handmade Electroporation Materials and Apparatus Setup (Prepare the Following in Advance of Electroporation Experiments):}

1. For DNA injection pipettes: pull DNA injection pipettes using a horizontal micropipette puller. Injection pipettes should have a fine tip with an opening less than $10 \mu \mathrm{m}$ to avoid tissue damage when injecting DNA into the embryonic cavities.

2. For glass capillary electroporation: use a microforge to cut the opening of DNA injection pipettes to an internal diameter of either 20 or $30 \mu m$. To avoid cellular damage when the capillary is in contact with the embryo for electroporation, the tip of the glass capillary must be cut cleanly and not contain sharp, broken edges.

3. For handmade capillary electrode (anode) (Figure 1): insert a $0.2 \mathrm{~mm}$ diameter platinum wire into an electroporation glass capillary with a fixed opening of 20 or $30 \mu \mathrm{m}$ diameter to focus the electric current and deliver the plasmid DNA to a small region of interest in the embryo.

4. For handmade L-shaped electrode (cathode) (Figure 1): bend a $0.2 \mathrm{~mm}$ diameter platinum wire creating an "L" shape, with the horizontal part of the "L" around $1 \mathrm{~mm}$ in length.

5. Attach each platinum electrode to a thin insulated wire and insert it into a microinjection needle holder covered by insulation tape.

6. Mount the needle holders on standard micromanipulation instrument holders.

7. Connect the circuit as shown in Figure 1: connect the capillary electrode to the anode of the power supply; connect the L-shaped electrode to the anode of the multimeter; connect the cathode of the mulimeter to the cathode of the power supply.

\section{Electroporation E7.5 or E8.5 Mouse Embryos}

1. Fill the electroporation glass capillary with PBS to within 1-2 mm of the top and insert the straight platinum electrode (anode) into the glass capillary until it reaches the bottom of the capillary.

2. Anchor the L-shaped electrode (cathode) on the surface of a $30 \mathrm{~mm}$ petri dish filled with PBS.

3. Transfer the embryo from M2 medium into the PBS-filled electroporation dish.

4. Insert the injection needle from lateral epiblast into the amniotic cavity of the embryo. Using a pneumatic pico pump, inject DNA solution (pCAG-Cre:GFP or pCAG-GFP $1-1.5 \mu \mathrm{g} / \mathrm{ml}$ with $0.01 \%$ green food coloring dye) into the cavity until it is completely full. For E7.5-E8.5 embryos, less than $5 \mu$ DNA solution is required for one embryo. Using the green dye as an indicator, be careful not to burst the embryo

5. Carefully position the embryo between the electrodes and move the capillary electrode to the precise location where the DNA is to be delivered.

Note: The orientation of the embryo depends on the region where the DNA is to be electroporated.

6. Electroporate the embryo using 200 volts $(\mathrm{V})$ in 6 pulses, each of 50 ms duration with a $1 \mathrm{sec}$ interval between each pulse.

7. Transfer the embryo to pre-equilibrated culture medium immediately after electroporation. If desired, repeat the process for the next embryo. Note: Add the culture medium into a sterile container and put it in the culture incubator to pre-equilibrate the medium.

8. To detect electroporated cells $2 \mathrm{hr}$ after culture, transfer the embryos to a clean $30 \mathrm{~mm}$ Petri dish of M2 medium using a pastette. Image the embryos as in step 5.9 using a fluorescence compound dissecting microscope.

9. Transfer the embryos back to culture using a pastette immediately after imaging.

10. To detect dead cells caused by electroporation, stain the embryos with a far-red cell membrane-impermeable nuclear dye (1:200 in embryo culture medium) at $37^{\circ} \mathrm{C}$ for $10 \mathrm{~min} 2 \mathrm{hr}$ after electroporation (Optional)

11. To count the electroporated cells, fix the embryos in $4 \%$ paraformaldehyde (PFA) for $2-4 \mathrm{hr}$ at $4{ }^{\circ} \mathrm{C}$, stain the nucleus with an ultraviolet or farred fluorescent nuclear counterstain and image the embryos using a confocal microscope (Optional).

Note: Embryonic growth is adversely affected if left too long in PBS. Therefore, ensure that the time taken to electroporate each embryo is minimized (<5 min per embryo).

\section{Representative Results}

\section{Grafting}

EpiSCs that ubiquitously express EGFP (r04-GFP, derived from E6.5 epiblast, and C2, derived in vitro from mESCs) ${ }^{4}$ were manually scraped from the culture dish and grafted into different sites of E7.5 embryos (Figure 2A). The embryos were cultured ex vivo and analysed after 24 hr. The distribution of donor cells was assessed by fluorescence microscopy. If donor cells incorporated, they proliferated and their derivatives dispersed within the host embryos (Figure 2B). It has been observed that grafts containing 10-16 cells incorporated efficiently in the host embryos (Figure 2A, and 2B), however, grafting more cells does not result in better chimaerism. Instead, grafted cells produced unincorporated clumps (Figure 2C and 2D).

\section{Electroporation}

To assess the efficiency of our electroporation system, we delivered GFP-expressing plasmids (pCAG-GFP and pCAG-Cre:GFP) to specific sites in the embryo. Consistent with a previous study ${ }^{11}, \mathrm{GFP}^{+}$cells were detected in embryos 1-2 hr after electroporation (Figure 2E and 2G). When distal epiblast cells at the late primitive streak stage embryo were electroporated, labelled cells contributed to the neural ectoderm after $24 \mathrm{hr}$ in culture (Figure 2E and 2F). This result corresponds well to known fate maps of epiblast cells in gastrulation stage embryos ${ }^{17}$. Similarly, when the GFP expression plasmid was electroporated in the primitive streak at E8.5 (2-5 somites), GFP ${ }^{+}$cells contributed to the paraxial mesoderm (Figure $2 \mathrm{G}$ and $2 \mathrm{H}$ ), consistent with known fate maps of the late primitive streak ${ }^{18}$. Moreover, we observed contribution to all three germ layers from electroporated cells (Figure 2I-K), suggesting that the electroporation procedure does not compromise cell behaviour in vivo. However, we 
also noticed that whilst epiblast (E7.5) or primitive streak cells (E8.5) were targeted, some endoderm cells were also electroporated (Figure 3C and Table 1).

One of the major advantages of using a capillary electrode is that the number of electroporated cells can be controlled, simply by changing the diameter of its opening. To determine the number of electroporated cells, embryos were fixed $2 \mathrm{hr}$ after electroporation and imaged in wholemount on a confocal microscope. The number of GFP ${ }^{+}$cells was manually counted in the confocal z-stacks. Table 1 shows that, for a given stage, increasing the opening size of the glass capillary from 20 to $30 \mu \mathrm{m}$ results in DNA uptake by more cells. When a single opening size was compared between stages (E7.5 versus E8.5), more cells were found to be electroporated at the latter stage. This effect may be due to a higher concentration of DNA present in the amniotic cavity at E8.5. Because the DNA solution was mixed with the green food dye, we can use the green color to assess the DNA concentration in the amniotic cavity. In the microscope, it is clear that, when compared with E8.5 embryos, the green color after DNA injection is much lighter in the cavity of E7.5 embryos. Although the same concentration of DNA solution was injected into E7.5 and E8.5 embryos, more DNA solution was in the amniotic cavity of E8.5 embryos in order to fill it completely because they are larger in the size. After withdrawing the injection needle, there is always some degree of leakage of DNA solution from the amniotic cavity, and since the puncture hole is larger in comparison to the size of the amniotic cavity in earlier embryos, it is likely that there was proportionately more leakage from E7.5 than E8.5 embryos, leading to a lower DNA concentration. The different number of transfected cells could also be due to the different diameters or induced transmembrane voltage (ITV) thresholds of cells at different stages.

A drawback of electroporation is the associated cell death. Similar to the traditional gold plated or needle-shaped electrodes, electroporation using a capillary electrode also causes the cell death. After electroporation the targeted region appeared darker in colour compared to neighbouring regions (Figure 3A and 3B), indicating that some degree of cell death must have occurred in this area. To further determine the number of dead cells caused by the electroporation procedure, embryos were stained with a fluorescent cell membrane-impermeable nuclear dye. The nuclei of dead cells were labelled with a membrane-impermeable far-red fluorescence dye. The staining confirmed that this capillary electroporation technique only results in a small number of dead cells near the electroporation site (Figure 3D and Table 1).

We noticed that although dead cells appear at the electroporation site, GFP ${ }^{+}$cells and dead cells are also most exclusive from each other (Figure 3E and 3F). Moreover, when the caudal lateral epiblast at E8.5 was electroporated with pCAG-GFP and a glass capillary opening of $20 \mu \mathrm{m}$, a large number of $\mathrm{GFP}^{+}$cells was detected after $48 \mathrm{hr}$ in culture (Figure $\mathbf{3 G}$ and $\mathbf{3 H}$ ). Taken together, these results suggest most GFP ${ }^{+}$ cells detected $2 \mathrm{hr}$ after electroporation are still viable during the further culture.

We scored the number of $\mathrm{GFP}^{+}$cells after $24 \mathrm{hr}$ ex vivo culture. Six embryos were electroporated with pCAG-GFP at E7.5, using a capillary opening of $20 \mu \mathrm{m}$ diameter. $107 \pm 31$ (mean \pm s.d.) GFP ${ }^{+}$cells/embryo were detected. Since at the start of culture ( $2 \mathrm{hr}$ ), 9 cells were electroporated on average per embryo (Table 1), this suggests that electroporated cells underwent 3-4 divisions within $2 \mathrm{hr}$. The average cell doubling time from E7.5 to E8.5 embryos is around 6-7 hr in all cells apart from those in the ventral node ${ }^{19,20}$. This suggests that the electroporation procedure does not hamper normal cell growth.

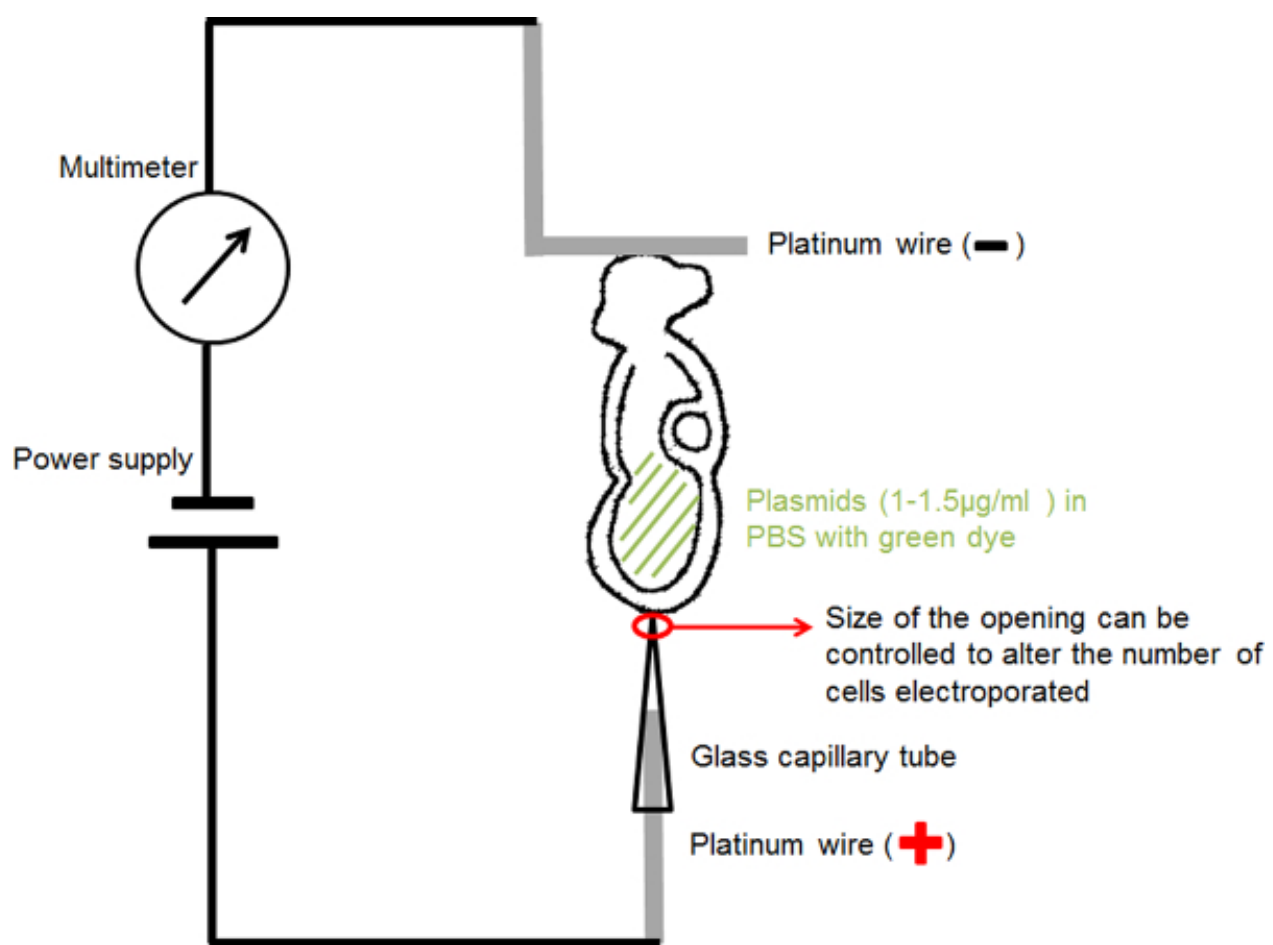

Figure 1. Circuit diagram showing the electroporation setup. The embryo containing DNA solution in its amniotic cavity was positioned between the two electrodes. Current at the chosen parameters was provided by a square wave pulse generator (power supply). A multimeter was connected in series to detect the electric current passing the embryo. Please click here to view a larger version of this figure. 

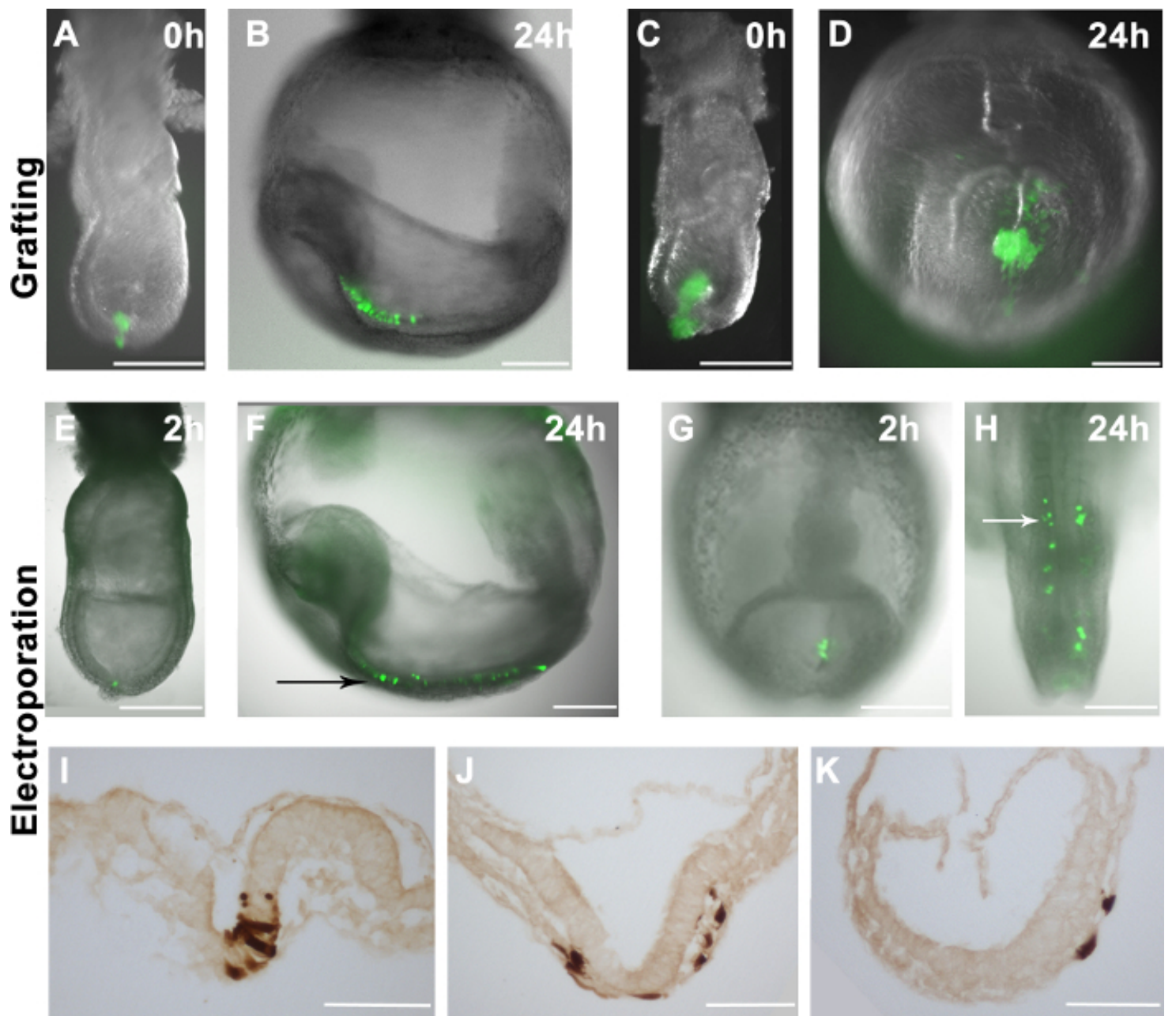

Figure 2. The distribution of grafted or electroporated cells in host embryos. (A-H) GFP fluorescence overlays (green) on brightfield images of wholemount embryos (grayscale) (A) 10-16 GFP ${ }^{+}$EpiSCs were grafted into the distal region of a late-streak stage embryo. (C) A larger clump of GFP ${ }^{+}$EpiSCs was grafted into the distal region of a mid-streak stage embryo. (B and $\left.\mathbf{D}\right)$ The distribution of EpiSCs derived cells (green) in the host embryos (shown in A and C) after $24 \mathrm{hr}$ in culture. (B) GFP ${ }^{+}$cells dispersed in the host embryo, suggesting correct integration of the donor cells. (D) Grafting larger cell clumps resulted in unincorporated clump formation in the host embryo. (E-K) pCAG-Cre:GFP plasmid electroporated into specific areas of wildtype embryos. Electroporating the distal region of an early bud stage embryo (E) or the primitive streak of a 2-5 somite stage embryo (G) resulted in $\mathrm{GFP}^{+}$cells in these regions $2 \mathrm{hr}$ after the procedure. (F and $\mathbf{H}$ ) The distribution of GFP cells in the host embryos after $24 \mathrm{hr}$ in culture, showing that electroporated cells contribute to the neuroectoderm (black arrow) (F) and paraxial mesoderm (white arrow) (H). (I-K) DAB immunostaining for the $\mathrm{GFP}^{+}$cells showing that the electroporated cells can give rise to the neuroectoderm (I), mesoderm $(\mathrm{J})$ and endoderm $(\mathrm{J}$ and $\mathrm{K})$ after $24 \mathrm{hr}$ in culture. Scale bar $(\mathrm{A}-\mathrm{H})=250 \mu \mathrm{m}$; scale bar $(\mathrm{I}-\mathrm{K})=100 \mu \mathrm{m}$. Note: Figure $1 \mathrm{~A}$ and $1 \mathrm{~B}$ are reprinted from our previous publication ${ }^{4}$. Please click here to view a larger version of this figure. 

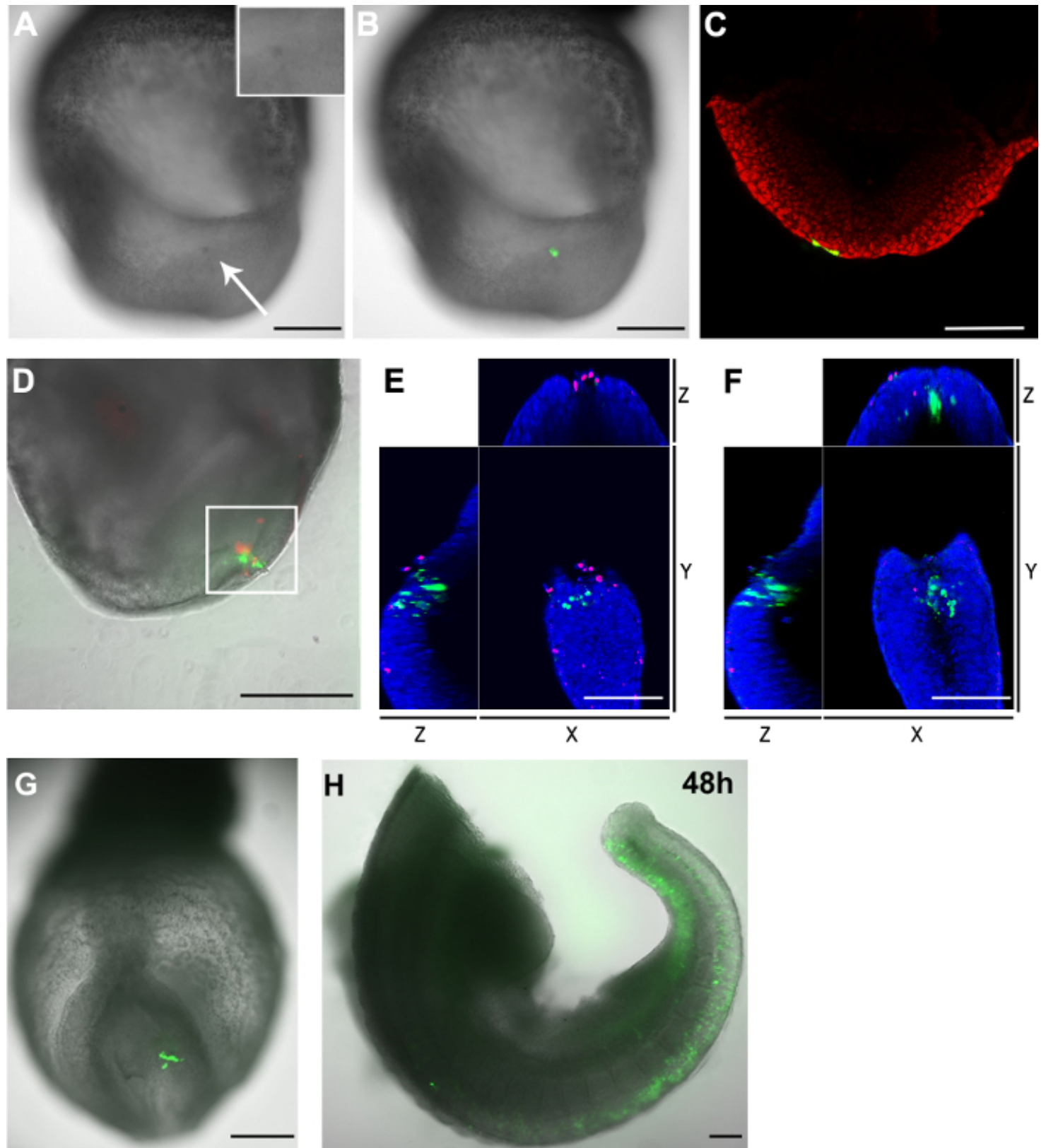

Figure 3. Distribution of GFP ${ }^{+}$cells and dead cells in the embryos after electroporation. (A-C) pCAG-Cre:GFP plasmid electroporated into the caudal lateral epiblast cells of an E8.5 (2-5 somite stage) embryo (capillary opening size: $20 \mu \mathrm{m}$ ). (A) $2 \mathrm{hr}$ after the procedure, the targeted region showed a dark color (white arrow) compared to other parts of the embryo. Inset shows an enlargement of the electroporated region. (B) Brightfield image (grayscale) overlaid with the green fluorescent channel showing the electroporated cells (green). (C) A confocal z-slice showing that two endoderm cells (green) also took up the plasmid when the caudal lateral epiblast cells were targeted. The cell nuclei are shown in red. (D-F) pCAG-Cre:GFP plasmid was electroporated in the caudal aspect of the node of an E8.5 embryo (capillary opening size: $30 \mu \mathrm{m}$ ). The embryo was cultured for $2 \mathrm{hr}$. Electroporated cells are shown in green and dead cells in red. (D) The electroporated area contains both GFP ${ }^{+}$ cells as well as dead cells. The area in the white box was further analyzed in a confocal microscope. Manual counting of the $z$-stack showed that there were $33 \mathrm{GFP}^{+}$cells and 23 dead cells in this area. Only two cells were both positive for both fluorophores. (E and F) XYZ view of a confocal z-slice from the white boxed region in D showing GFP $^{+}$cells are separate from the dead cells. The nuclei are shown in blue. (G and $\left.\mathbf{H}\right)$ pCAG-Cre:GFP plasmid was electroporated into a few cells in the caudal lateral epiblast of an E8.5 embryo (capillary opening size: $20 \mu \mathrm{m}$ ) and imaged after two $(\mathbf{G})$ and $48(\mathbf{H})$ hours ex vivo culture Note: $(\mathrm{H})$ The embryo were severed in two after culture. The head and heart regions were removed. Scale bar $(A, B, D, G$ and $H)=250 \mu \mathrm{m}$; scale bar $(C, E$ and $F)=100 \mu \mathrm{m}$. Please click here to view a larger version of this figure. 


\begin{tabular}{|c|c|c|c|c|}
\hline $\begin{array}{l}\text { Diameter of opening of the } \\
\text { capillary tube }\end{array}$ & Embryo stage & $\begin{array}{l}\text { Electroporation efficiency: } \\
\text { no. embryos containing } \\
\text { GFP }^{+} \text {cells after } 2 \mathrm{~h} / \\
\text { total no. of electroporated } \\
\text { embryos (no. GFP }{ }^{+} \\
\text {embryos that developed } \\
\text { normally after } 24 \text { or } 48 \mathrm{~h} \\
\text { culture) }\end{array}$ & $\begin{array}{l}\text { Average number of GFP } \\
\text { cells per embryo } \pm \text { s.d. }(n= \\
\text { no. of examined embryos) }\end{array}$ & $\begin{array}{l}\text { No. of GFP }{ }^{+} \text {endodermal } \\
\text { cells per each embryo } \\
\pm \text { s.d. ( } n=\text { no. of examined } \\
\text { embryos) }\end{array}$ \\
\hline $20 \mu \mathrm{m}$ & E7.5 (LS-LB) & $7 / 9(7)$ & $9 \pm 3(n=4)$ & $4 \pm 2(n=4)$ \\
\hline $30 \mu \mathrm{m}$ & E7.5 (LS-LB) & $13 / 15(12)$ & $17 \pm 2(n=4)$ & $6 \pm 1(n=4)$ \\
\hline $20 \mu \mathrm{m}$ & E8.5 (2-5 somites) & $12 / 13(10)$ & $21 \pm 4(n=4)$ & $11 \pm 4(n=4)$ \\
\hline $30 \mu \mathrm{m}$ & E8.5 (2-5 somites) & $2 / 2(2)$ & 33 and $26(n=2)$ & 14 and $16(n=2)$ \\
\hline
\end{tabular}

Table 1. Electroporation efficiency of pCAG-Cre:GFP plasmid in mouse embryos.

Abbreviation: LS, late primitive streak stage; LB: late bud stage. Embryos are staged according to Downs and Davies ${ }^{12}$

\section{Discussion}

\section{Grafting}

The critical step for cell grafting experiments is the insertion of a coherent string of cells ideally in a single action, to avoid breakup of the clump. This technique requires some practice in controlling the mouth pipette. If donor cells incorporate well in the host, their derivatives will disperse in the embryo. To further determine whether the dispersed donor derived-cells differentiate appropriately in the host, immunostaining can be performed on the embryo sections. If donor cells are not compatible with the host environment, they either cannot be detected (as they are expelled from the embryo) or form unincorporated clumps in the embryos after culture. If both dispersed cells and cell clumps were observed, this may indicate that too many cells were grafted and excessive donor cells which cannot interact with surrounding host cells resulted in clump formation. In this case, additional grafts containing a smaller number of cells can be performed.

The major limitation of the cell grafting technique is that it is not possible to determine the full in vivo potential of cells since mouse ex vivo culture over periods longer than $48 \mathrm{hr}$ has not been achieved. However, if combined with ultrasound-guided cell injection, it may be possible to transfer cultured cells to the embryos in utero. To summarize, cell grafting experiments have been widely used in our group and have given us valuable clues about the in vivo potential of various cell types ${ }^{4,21,22}$. It is a technique of general utility to assess the in vivo potential of in vitro cultured cells in early postimplantation embryos.

\section{Electroporation}

Although in this study we have only shown that it is efficient to use the capillary electroporation technique to target the epiblast, it is also possible to intentionally target other germ layers such as endoderm cells. The critical step for the capillary electroporation technique is to minimize the time taken to electroporate each embryo (<5 min per embryo) since PBS is very suboptimal for early mouse embryos. Our data above has shown that, in most areas in the embryos, electroporation does not affect the embryo growth. However, electroporation in the node caused abnormal development and led to the premature death of the embryo. This is likely due to damage or death of the cells that form important signalling centres ${ }^{23}$. Hence, this region would have to be avoided with this technique. A further caveat is that, as mentioned in the results section, whilst epiblast or primitive streak cells were targeted, some endoderm cells were also electroporated. This may be because DNA reaches to the endoderm through gaps under the epiblast epithelium. Endoderm is composed of epithelial cells and in our experience these cells have a higher propensity to take up DNA. Therefore, when applying this technique for fate mapping, it is important to assess which cells initially take up DNA.

It should also be noted that although pCAG-GFP and pCAG-Cre:GFP plasmids can be efficiently delivered using the electroporation parameters shown in this study, the efficiency of other DNA constructs may vary and need individual optimisation. Alterations in DNA concentration, electroporation voltage or the number of pulses can be made if plasmids are found to be difficult to transfect.

To summarize, our optimized capillary electroporation system can efficiently and reproducibly deliver GFP or Cre:GFP plasmids into a very few cells in the embryo with limited cell death. Since this method does not require expensive or highly specialized equipment, it can be of great use for cell tracking studies or in testing the effect of ectopic expression or conditional deletion of genes in early embryos, if electroporation is performed in embryos carrying floxed conditional mutant alleles. Therefore, this electroporation technique provides a useful functional tool for understanding on a cell-by-cell basis the roles of cell-intrinsic factors in the context of localized wildtype embryonic environments.

\section{Disclosures}

The authors have no conflicts of financial or other interest to declare.

\section{Acknowledgements}

We thank Filip Wymeersch and Anestis Tsakiridis for comments on the manuscript, staff in the SCRM animal unit for help with animal maintenance and Prof. Stuart Forbes for immunohistochemistry reagents. This work was supported by MRC grant Mr/K011200/1 and the China Scholarship Council 


\section{References}

1. O'Hagan, A. R., Morton, R., \& Eid, N. Loss of asthma control in pediatric patients after discontinuation of long-acting Beta-agonists. Pulmonary med. 2012, 894063 (2012).

2. Brons, I. G. et al. Derivation of pluripotent epiblast stem cells from mammalian embryos. Nature. 448, 191-195 (2007).

3. Tesar, P. J. et al. New cell lines from mouse epiblast share defining features with human embryonic stem cells. Nature. 448, 196-199 (2007).

4. Huang, Y., Osorno, R., Tsakiridis, A., \& Wilson, V. In Vivo differentiation potential of epiblast stem cells revealed by chimeric embryo formation. Cell rep. 2, 1571-1578 (2012).

5. Sadik, M. M. et al. Scaling relationship and optimization of double-pulse electroporation.Biophys. J. 106, 801-812 (2014).

6. Kaestner, L., Scholz, A., \& Lipp, P. Conceptual and technical aspects of transfection and gene delivery. Bioorg. Med. Chem. Lett. (2015).

7. Soares, M. L., Torres-Padilla, M. E., \& Zernicka-Goetz, M. Bone morphogenetic protein 4 signaling regulates development of the anterior visceral endoderm in the mouse embryo. Dev. Growth Differ. 50, 615-621 (2008).

8. Pierreux, C. E., Poll, A. V., Jacquemin, P., Lemaigre, F. P., \& Rousseau, G. G. Gene transfer into mouse prepancreatic endoderm by whole embryo electroporation. JOP. 6, 128-135 (2005).

9. Falk, J. et al. Electroporation of cDNA/Morpholinos to targeted areas of embryonic CNS in Xenopus. BMC Dev. Biol. 7, 107 (2007).

10. Davidson, B. P., Tsang, T. E., Khoo, P. L., Gad, J. M., \& Tam, P. P. Introduction of cell markers into germ layer tissues of the mouse gastrula by whole embryo electroporation. Genesis. 35, 57-62 (2003).

11. Khoo, P. L., Franklin, V. J., \& Tam, P. P. Fate-Mapping Technique: Targeted Whole-Embryo Electroporation of DNA Constructs into the Germ Layers of Mouse Embryos 7-7.5 Days Post-coitum. CSH protocols. 2007, pdb.prot4893 (2007).

12. Tawk, M., Bianco, I. H., \& Clarke, J. D. Focal electroporation in zebrafish embryos and larvae. Methods Mol Biol. 546., 145-151 (2009).

13. Haas, K., Jensen, K., Sin, W. C., Foa, L., \& Cline, H. T. Targeted electroporation in Xenopus tadpoles in vivo--from single cells to the entire brain. Differentiation. 70, 148-154 (2002).

14. Nolkrantz, K. et al. Electroporation of single cells and tissues with an electrolyte-filled capillary. Anal. Chem. 73, 4469-4477 (2001).

15. Mazari, E. et al. A microdevice to locally electroporate embryos with high efficiency and reduced cell damage. Development. 141, 2349-2359 (2014).

16. Copp, A. J., \& Cockroft, D. L. Postimplantation mammalian embryos : a practical approach. IRL Press. (1990).

17. Tam, P. P., \& Behringer, R. R. Mouse gastrulation: the formation of a mammalian body plan. Mech. Dev. 68, 3-25 (1997).

18. Wilson, V., \& Beddington, R. S. Cell fate and morphogenetic movement in the late mouse primitive streak. Mech. Dev. 55., 79-89 (1996).

19. Tzouanacou, E., Wegener, A., Wymeersch, F. J., Wilson, V., \& Nicolas, J. F. Redefining the progression of lineage segregations during mammalian embryogenesis by clonal analysis. Dev Cell. 17, 365-376 (2009).

20. Bellomo, D., Lander, A., Harragan, I., \& Brown, N. A. Cell proliferation in mammalian gastrulation: the ventral node and notochord are relatively quiescent. Dev. Dynam. 205., 471-485 (1996).

21. Tsakiridis, A. et al. Distinct Wnt-driven primitive streak-like populations reflect in vivo lineage precursors. Development. 141, 1209-1221 (2014).

22. Gouti, M. et al. In vitro generation of neuromesodermal progenitors reveals distinct roles for wnt signalling in the specification of spinal cord and paraxial mesoderm identity. PLoS biology. 12, e1001937 (2014).

23. Beddington, R. S. Induction of a second neural axis by the mouse node. Development. 120, 613-620 (1994). 